\title{
Nimotuzumab with Concurrent Chemo-Radiotherapy in Patients with Locally Advanced Squamous Cell Carcinoma of Head and Neck (LASCCHN)
}

\author{
Naresh Somani \\ Department of Clinical Trial and Research, Bhagwan Mahaveer Cancer Hospital and Research Centre, \\ Jaipur, India \\ Email: drsomani@cancerindia.com
}

Received 9 March 2015; accepted 13 April 2015; published 22 April 2015

Copyright (C) 2015 by authors and Scientific Research Publishing Inc.

This work is licensed under the Creative Commons Attribution International License (CC BY). http://creativecommons.org/licenses/by/4.0/

(c) () Open Access

\section{Abstract}

Background: Head and neck cancers (HNCs) constitute 5\% of all cancers globally and are the most common cancers in India. Chemotherapy and radiotherapy have not been proved to be effective in advanced cases and the prognosis remains dismal. This underscores the need for newer treatment options in these cases. Nimotuzumab, an anti-epidermal growth factor receptor (anti-EGFR) monoclonal antibody, was safer when combined with chemo- or radio-therapy. Aim: To evaluate the safety and efficacy of concurrently administered nimotuzumab with chemo-radiotherapy in patients with advanced inoperable squamous cell carcinomas of head and neck (LASCCHN). Methods: This was an open-label, single arm study evaluating 57 patients with histologically confirmed inoperable LASCCHN (stages III and IV) and eastern co-operative oncology group (ECOG) performance status $<2$. Informed consent was obtained from all patients. The patients were administered IV cisplatin $30 \mathrm{mg} / \mathrm{m}^{2}$ and IV nimotuzumab $200 \mathrm{mg}$ weekly for 6 weeks, along with radiotherapy of 6600 cGy over 33 fractions. Patients were evaluated over response evaluation criteria in solid tumors (RECIST) criteria 24 weeks after the last cycle of chemotherapy. Results: Mean age of patient was 50 years old ( 29 - 79 years old). The most common site of cancer was oral cavity (56.1\%). Forty six patients $(80.7 \%)$ completed 6 cycles of therapy. Objective response rate (ORR) was $80.7 \%$, with 34 patients (59.6\%) achieving complete response (CR), and 12 (21\%) achieving partial response (PR). Stable disease (SD) was noted in $8(14 \%)$ patients and progressive disease in $3(5.2 \%)$ patients. Conclusion: Addition of nimotuzumab is a safe and efficacious option in patients with inoperable LASCCHN. Our observations confirm the available Phase II data. The long term survival benefits based on this encouraging response rate need to be further evaluated in this subset of cancer patients. 


\section{Keywords}

\section{Nimotuzumab, Anti-Epidermal Growth Factor Receptor (Anti-EGFR), Chemotherapy, Radiotherapy, Locally Advanced Squamous Cell Carcinoma of Head and Neck (LASCCHN)}

\section{Introduction}

Ranking $6^{\text {th }}$, head and neck cancers (HNCs) constitute approximately $5 \%$ of all cancers globally [1], and are the most common cancers in India, with an incidence of approximately $0.2-0.25$ million cases annually [2]. Out of all head and neck cancers occurring globally, 57.5\% occur in Asia especially in India. Incidence is higher in South Asian people and in the USA, incidence rates are two-fold higher in Blacks compared to Whites. Of all the malignant tumours of the head and neck region, squamous cell carcinoma (SCC) is the most frequent. The most important risk factors are tobacco exposure, either by smoking or chewing, alcohol consumption, and infection with high-risk types of human papillomavirus [3]. In India, tobacco chewing accounts for nearly $50 \%$ of oral and oropharyngeal tumours in men and over $90 \%$ in women. In addition, it has also been associated with ionizing radiation, diesel exhausts, sulphuric acid mists, and mustard gas [4].

There is an increased likelihood of SCC developing from preneoplastic lesions grouped under the term dysplasia. The clinical features of HNC vary with the site of the tumour. For example, patients with tumours arising in nasal and paranasal sinuses present with nasal fullness, stuffiness, or obstruction, epistaxis, rhinorrhea, pain, and paraesthesia. Patients with nasopharyngeal carcinoma present with painless enlargement of upper cervical lymph nodes with a blood-stained post-nasal drip. Tumours of the hypopharynx, larynx or trachea, may present with fluctuating hoarseness, sore throat, and/or chronic cough of a few months' duration [4].

A dysplastic lesion may be self-limiting or may even turn into SCC. Malignant transformation can occur in $11 \%$ moderate to severe dysplastic lesions. Surgery and/or radiotherapy (RT) provide favourable outcome in the most early-stage tumours. In advanced tumours, survival outcomes are poor ( $40 \%-50 \%$ five-year survival rates) [4].

Radiotherapy (RT) is the standard-of-care in initial stages of HNC, while addition of chemotherapy, especially cisplatin, is warranted in non-resectable and locally advanced cases of squamous cell carcinoma of the head and neck (LASCCHN). However, results have not been proved to be effective in advanced cases, and the prognosis remains dismal. This underscores the need for newer treatment options in these cases. At the forefront of research are therapies involving molecular targets such as epidermal growth factor receptor (EGFR), a topic have extensively researched over the last decade. Overexpression of EGFR has been observed in various cancers including gliomas [5], sarcomas [6] and HNCs [7]. In SCCs of head and neck, EGFR is over-expressed in $>90 \%$ patients [7].

Nimotuzumab (also known as h-R3), is a humanized monoclonal antibody that recognizes the EGFR external domain (domain III) with intermediate affinity, and has demonstrated a remarkable anti-proliferative, pro-apoptotic and anti-angiogenic effect in preclinical in vitro as well as in vivo studies. It has also demonstrated good safety profile in combination with chemo-radiotherapy (CRT) as compared to other monoclonal antibodies [8] [9].

This study was conducted with the aim of further evaluating the safety and efficacy of concurrently administering Nimotuzumab with chemo-radiotherapy in patients with LASCCHN.

\section{Methods}

This was an open-label, single-arm study. Patients above the age of 18 years, having histologically confirmed LASCCHN, in an inoperable stage (III and IV), were enrolled. Other main inclusion criteria were Eastern Cooperative Oncology Group (ECOG) Performance Status score of $\leq 2$ and life expectancy greater than 6 months. The main exclusion criteria were patients who received chemotherapy, radiotherapy and/or immunotherapy, patients with distant metastases and known or suspected hypersensitivity to drugs used in the study. Informed consent was obtained from all patients.

Interventions: Baseline hematological and biochemical profiles were done before starting the treatment. Radiotherapy schedule was total dose 6600 cGy, 200 cGy/fraction, 5 fractions/wk for total of 33 fractions. For che- 
motherapy, cisplatin was given in a dose of $30 \mathrm{mg} / \mathrm{m}^{2}$ weekly for 6 weeks. Study drug nimotuzumab was given by 60 mins I.V. infusion $200 \mathrm{mg}$ weekly for 6 weeks. The patients were evaluated every week during the treatment period and there after till the complete clearance of reactions. Patients were evaluated over response evaluation criteria in solid tumors (RECIST) criteria 24 weeks after the last cycle of chemotherapy. Toxicity was documented, using radiation therapy oncology group (RTOG) acute toxicity criteria. For efficacy, intent-to-treat (ITT) analysis was performed following the last-observation-carried-forward (LOCF) principle. Data were evaluated using a Chi-square test and Fisher's exact probability test as appropriate. Median overall survival along with 95\% CI, mean, and the standard error was estimated by the Kaplan-Meier method.

\section{Results}

\subsection{Demographics}

Mean age of patients was 50 years old, with majority of them (56.1\%) between the age of 40 and 60 years old (Table 1). The most common site of cancer was oral cavity (56.1\%) (Table 2). Forty-six patients (80.7\%) completed 6 cycles of therapy. Majority of the patients (77\%) were males.

\subsection{Efficacy and Safety Result}

Objective response rate (ORR) was 80.7\%, with 34 patients (59.6\%) achieving complete response (CR), and 12 (21\%) achieving partial response (PR). Stable disease (SD) was noted in 8 (14\%) patients and progressive disease in $3(5.2 \%)$ patients (Figure 1).

The common adverse event observed in the study is mucositis, seen in 19 patients (33\%). No Grade III or IV adverse events were reported. Nimotuzumab did not exacerbate adverse events associated with concurrent CRT.

\section{Discussion}

Nimotuzumab has been extensively evaluated and studied in clinical and non-clinical trials with encouraging results [10]-[12]. The results of this study were comparable with other studies conducted in the past with similar combination [8]. On the other hand, even though cetuximab was found to improve overall survival at 5 years in

Table 1. Distribution of patients with respect to age.

\begin{tabular}{cc}
\hline Age (Years Old) & No. of Subjects \\
\hline $20-30$ & 5 \\
$31-40$ & 6 \\
$41-50$ & 18 \\
$51-60$ & 14 \\
$61-70$ & 11 \\
$>70$ & 3 \\
Total & $\mathbf{5 7}$ \\
Mean Age & $\mathbf{5 0}$ \\
\hline
\end{tabular}

Table 2. Distribution of patients with respect to tumour location.

\begin{tabular}{cccc}
\hline Tumour Location & Male & Female & Total \\
\hline Oropharynx & 5 & 3 & 8 \\
Oral Cavity & 27 & 5 & 32 \\
Hypopharynx & 12 & 5 & 17 \\
Total & 44 & 13 & 57 \\
\hline
\end{tabular}




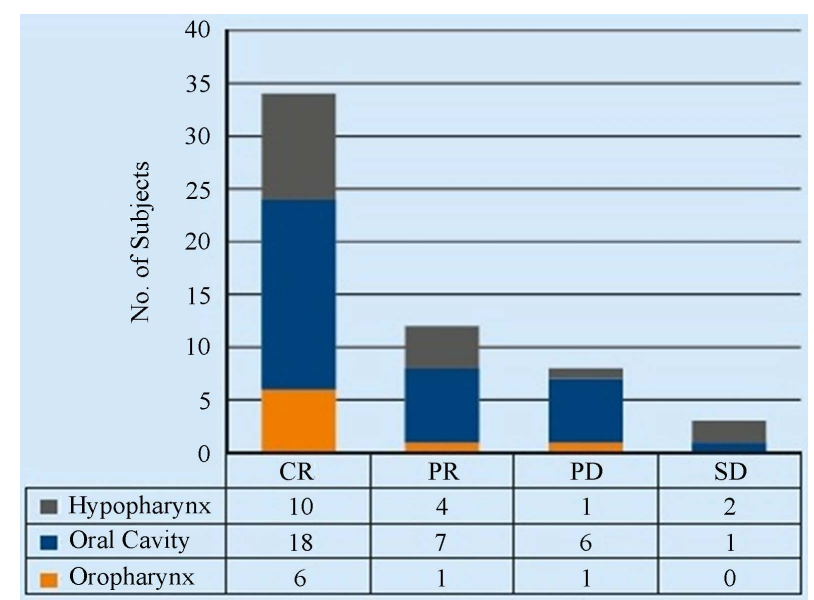

CR: complete response; PR: partial response; PD: progressive disease; SD: Stable disease.

Figure 1. Response rate with respect to tumour location.

a study [13], it was also found to induce moderate to severe rash in almost $87 \%$ patients in another study [14]. Other monoclonal antibodies, including nimotuzumab, panitumumab and zalitumumab are being evaluated for enhanced properties like antibody-dependent cell-mediated cytotoxicity and unique binding sites [15].

Alternatively, tyrosine kinase inhibitors do not induce antibody-dependent cell-mediated cytotoxicity and may be less efficacious than monoclonal antibodies due to the lack of tyrosine kinase mutations in this cancer [16].

Overexpression of EGFR is known to cause radio-resistance in cells [9] [17], since EGFR and downstream Akt-signalling are known as key players in mediating cell survival following irradiation. It is therefore possible that anti-EGFR agents have a dual mode of action. First they inhibit the EGFR-signalling pathway and reduce the rate of cellular proliferation, and second they negate the EGFR-mediated radio-resistance and sensitize malignant cells to concomitant RT [18] [19].

Studies have also shown that RT and EGFR inhibitors may act additively or maybe even synergistically in promoting tumour cell death, which was suggested by the fact that pre-treatment of cells with EGFR inhibitors increased radiation-induced apoptosis by more than 3-fold [17] [18]. Similarly, EGFR antagonists may augment the tumour response to chemotherapy since EGFR overexpression is also known to cause resistance to chemotherapeutic agents [20].

As compared to other monoclonal antibodies, nimotuzumab has been proved to be remarkably safe repeatedly [8] [21] [22]. In total, nimotuzumab has been administered to more than 4000 patients and notably no evidence of severe skin rash has been reported. This safety profile can be attributed to the fact that unlike other anti-EGFR antibodies, nimotuzumab requires bivalent binding for stable attachment, leading to selective binding to cells expressing moderate to high EGFR levels. The monovalent Fab fragment of nimotuzumab has a ten-fold lower affinity for the extracellular domain of EGFR than the Fab of cetuximab. When EGFR density is low, such as in normal tissues, nimotuzumab monovalent interaction is transient, thus sparing healthy tissues and avoiding severe toxicities [22].

The limitations of this study include small sample size and incomplete capturing of the survival outcomes. We have not been able to stratify the patients based on EGFR expression levels and would contend to include it in our future studies.

Nimotuzumab being a humanized monoclonal antibody with optimized affinity constant has shown a versatile compatibility with the existing therapies, be it either radiotherapy or chemotherapy without any added toxicities. In our study no serious skin reactions have been recorded and it reflects the scenario in majority of patients receiving nimotuzumab. However, larger clinical trials are needed to prove its value.

\section{Conclusion}

Although inoperable LASCCHN is difficult to treat, new emerging therapies have shown to improve the overall outcome. While these continue to show promising results, detecting these cases early is also imperative to maximize positive results. The findings of this study support the use of nimotuzumab with CRT/RT as a viable the- 
rapeutic option in patients with inoperable LASCCHN. Even with these positive results, further validation of these findings is necessary with well-designed, large late stage clinical trials and extensive clinical experiences.

\section{Competing Interests}

The authors declare that they have no competing interests.

\section{References}

[1] Reuter, C.W., Morgan, M.A. and Eckardt, A. (2007) Targeting EGF-Receptor-Signalling in Squamous Cell Carcinomas of the Head and Neck. British Journal of Cancer, 96, 408-416. http://dx.doi.org/10.1038/sj.bjc.6603566

[2] Trivedi, N.P., Kekatpure, V.D., Trivedi, N.N. and Kuriakose, M.A. (2012) Head and Neck Cancer in India: Need to Formulate Uniform National Treatment Guideline. Indian Journal of Cancer, 49, 6-10. http://dx.doi.org/10.4103/0019-509X.98907

[3] Vermorken, J.B. and Specenier, P. (2010) Optimal Treatment for Recurrent/Metastatic Head and Neck Cancer. Annals Oncology, 21, vii252-vii261. http://dx.doi.org/10.1093/annonc/mdq453

[4] Rousseau, A. and Badoual, C. (2012) Head and Neck: Squamous Cell Carcinoma: An Overview. Atlas of Genetics and Cytogenetics in Oncology and Haematology, 16, 145-155. http://atlasgeneticsoncology.org/Tumors/HeadNeckSCCID5090.html

[5] Ekstrand, A.J., James, C.D., Cavenee, W.K., Seliger, B., Pettersson, R.F. and Collins, V.P. (1991) Genes for Epidermal Growth Factor Receptor, Transforming Growth Factor Alpha, and Epidermal Growth Factor and Their Expression in Human Gliomas in Vivo. Cancer Research, 51, 2164-2172.

[6] Little, S.E., Bax, D.A., Rodriguez-Pinilla, M., Natrajan, R., Messahel, B., Pritchard-Jones, K., et al. (2007) Multifaceted Dysregulation of the Epidermal Growth Factor Receptor Pathway in Clear Cell Sarcoma of the Kidney. Clinical Cancer Research, 1, 4360-4364. http://dx.doi.org/10.1158/1078-0432.CCR-07-0398

[7] Stanton, P., Richards, S., Reeves, J., Nikolic, M., Edington, K., Clark, L., et al. (1994) Epidermal Growth Factor Receptor Expression by Human Squamous Cell Carcinomas of the Head and Neck, Cell Lines and Xenografts. British Journal of Cancer, 70, 427-433. http://dx.doi.org/10.1038/bjc.1994.322

[8] Babu, K.G., Viswanath, L., Reddy, B.K., Shenoy, K., Shenoy, A., Naveen, T., et al. (2010) An Open-Label, Randomized, Study of h-R3mAb (Nimotuzumab) in Patients with Advanced (Stage III or IVa) Squamous Cell Carcinoma of Head and Neck (SCCHN): Four-Year Survival Results from a Phase IIb Study. Journal of Clinical Oncology, ASCO Annual Meeting Abstracts, 28, 5530.

[9] Bianco, C., Tortoram, G., Bianco, R., Caputo, R., Veneziani, B.M., Caputo, R., et al. (2002) Enhancement of Antitumor Activity of Ionizing Radiation by Combined Treatment with the Selective Epidermal Growth Factor Receptor-Tyrosine Kinase Inhibitor ZD1839 (Iressa). Clinical Cancer Research, 8, 3250-3258.

[10] Crombet, T., Osorio, M., Cruz, T., Roca, C., del Castillo, R., Mon, R., et al. (2004) Use of the Humanized Anti-Epidermal Growth Factor Receptor Monoclonal Antibody h-R3 in Combination with Radiotherapy in the Treatment of Locally Advanced Head and Neck Cancer Patients. Journal of Clinical Oncology, 22, 1646-1654. http://dx.doi.org/10.1200/JCO.2004.03.089

[11] Ramakrishnan, M., Eswaraiah, A., Crombet, T., Piedra, P., Saurez, G., Iyer, H. and Arvind, A.S. (2009) Nimotuzumab, a Promising Therapeutic Monoclonal for Treatment of Tumors of Epithelial Origin. mAbs, 1, 41-48. http://dx.doi.org/10.4161/mabs.1.1.7509

[12] Winquist, E., Nabid, A., Sicheri, A., Ganguly, P., Venkatesan, V., Schneider, K., et al. (2002) A Phase I Dose Escalation Study of a Humanized Monoclonal Antibody to EGFR (hR3) in Patients with Locally Advanced Squamous Cell Cancer of the Head and Neck (SCCHN) Treated with Radiotherapy (RT). Proceedings of American Society of Clinical Oncology, 21, 926.

[13] Bonner, J.A., Harari, P.M., Giralt, J., Cohen, R.B., Jones, C.U., Sur, R.K., et al. (2010) Radiotherapy Plus Cetuximab for Locoregionally Advanced Head and Neck Cancer: 5-Year Survival Data from a Phase 3 Randomised Trial, and Relation between Cetuximab-Induced Rash and Survival. The Lancet Oncology, 11, 21-28. http://dx.doi.org/10.1016/S1470-2045(09)70311-0

[14] Bonner, J.A., Harari, P.M., Giralt, J., Azarnia, N., Shin, D.M., Cohen, R.B., et al. (2006) Radiotherapy Plus Cetuximab for Squamous-Cell Carcinoma of the Head and Neck. New England Journal of Medicine, 354, 567-578. http://dx.doi.org/10.1056/NEJMoa053422

[15] Cassell, A. and Grandis, J.R. (2010) Investigational EGFR-Targeted Therapy in Head and Neck Squamous Cell Carcinoma. Expert Opinion on Investigational Drugs, 19, 709-722. http://dx.doi.org/10.1517/13543781003769844

[16] Li, H.F., Kim, J.S. and Waldman, T. (2009) Radiation-Induced Akt Activation Modulates Radioresistance in Human 
Glioblastoma Cells. Radiation Oncology, 4, 43. http://dx.doi.org/10.1186/1748-717X-4-43

[17] Wang, H.Q., Quan, T., He, T., Franke, T.F., Voorhees, J.J. and Fisher, G.J. (2003) Epidermal Growth Factor Receptor-Dependent, NF-kB-Independent Activation of the Phosphatidylinositol 3-Kinase/Akt Pathway Inhibits Ultraviolet Irradiation-Induced Caspases-3,-8, and -9 in Human Keratinocytes. The Journal of Biological Chemistry, 278, 4573745745. http://dx.doi.org/10.1074/jbc.M300574200

[18] Nagane, M., Levitzki, A., Gazit, A., Cavenee, W.K. and Huang, H.J. (1998) Drug Resistance of Human Glioblastoma Cells Conferred by a Tumor-Specific Mutant Epidermal Growth Factor Receptor through Modulation of Bcl- $\mathrm{X}_{\mathrm{L}}$ and Caspase-3-Like Proteases. Proceedings of the National Academy of Sciences of the United States of America, 95, 57245729. http://dx.doi.org/10.1073/pnas.95.10.5724

[19] Wang, X., McCullough, K.D., Franke, T.F. and Holbrook, N.J. (2000) Epidermal Growth Factor Receptor-Dependent Akt Activation by Oxidative Stress Enhances Cell Survival. The Journal of Biological Chemistry, 275, 14624-14631. http://dx.doi.org/10.1074/jbc.275.19.14624

[20] Rodriguez, M.O., Rivero, T.C., del Castillo Bahi, R., Muchuli, C.R., Bilbao, M.A., Vinageras, E.N., et al. (2010) Nimotuzumab Plus Radiotherapy for Unresectable Squamous-Cell Carcinoma of the Head and Neck. Cancer Biology \& Therapy, 9, 343-349. http://dx.doi.org/10.4161/cbt.9.5.10981

[21] Rojo, F., Gracias, E., Villena, N., Cruz, T., Corominas, J.M., Corradino, I., et al. (2010) Pharmacodynamic Trial of Nimotuzumab in Unresectable Squamous Cell Carcinoma of the Head and Neck: A SENDO Foundation Study. Clinical Cancer Research, 16, 2474-2482. http://dx.doi.org/10.1158/1078-0432.CCR-09-3042

[22] Garrido, G., Tikhomirov, I.A., Rabasa, A., Yang, E., Gracia, E., Iznaga, N., et al. (2011) Bivalent Binding by Intermediate Affinity of Nimotuzumab: A Contribution to Explain Antibody Clinical Profile. Cancer Biology \& Therapy, 11, 373-382. http://dx.doi.org/10.4161/cbt.11.4.14097 